\title{
Impacto do estadiamento radiológico na deteç̧ão de metástases ocultas do câncer de mama em estádios precoces
}

\author{
Acadêmicos: Mariana da Silva Vilas Boas, Victor Van Vaisberg \\ Orientadores: Gregório Pinheiro Soares, Max Senna Mano, Cheng Tzu Yen, Pedro Henrique \\ Isaacson Velho
}

Introdução: O Câncer de Mama é o segundo câncer mais frequente no mundo e o primeiro entre as mulheres. Metástases à distância são raras e podem ser encontradas em aproximadamente 4\% dos cânceres de mama ao estadiamento radiológico inicial (ER), de modo que a agência americana National Comprehensive Cancer Network (NCCN) recomenda apenas o ER guiado pela presença de sintomas, como dor óssea, ou alterações laboratoriais, como aumento de fosfatase alcalina. A American Society of Clinical Oncology's (ASCO) lista a eliminação do ER em pacientes com câncer de mama local com baixo risco para metástases uma das cinco ferramentas para otimizar custos qualidade dos cuidados ao paciente oncológico. Apesar destas evidências, o estadiamento radiológico para pacientes assintomáticos com câncer de mama precoce continua a ser amplamente empregada nos serviços especializados de cuidado ao câncer.

Objetivo: O objetivo deste estudo é avaliar as características clínicas e demográficas de pacientes recém diagnosticadas submetidas ao estadiamento radiológico em uma instituição terciária com alta demanda de casos de câncer de mama.

Métodos: Foi realizada uma análise retrospectiva dos casos de câncer de mama recentemente diagnosticados referenciados ao Instituto do Câncer do Estado de São Paulo entre 2010 e 2012. As seguintes variáveis foram analisadas: subtipos histológicos, estágios, frequência de estadiamento radiológico e informações sobre a presença de sintomas.

Resultados: De 1094 pacientes inicialmente selecionadas, foram analisados dados de 753 pacientes com estágio I e II de câncer de Mama de acordo com a sétima edição do American Joint Committee on Cancer (AJCC). A maioria dos tumores eram carcinomas ductais (83,5\%) e Estágio II (61,5\%). A maioria das pacientes (95,1\%) não havia relatado sintomas que poderiam indicar doença avançada. Não obstante, $90,8 \%$ foi submetida apelo menos um método radiológico. Doença metastática foi diagnosticada em 32 pacientes, sendo que 25 não apresentavam com nenhum sintoma relatado nem anormalidades em testes laboratoriais. 28 pacientes detectados com metástases $(87,5 \%)$ eram, inicialmente, fase II. O principal sítio de metástases foi o osso (62,5\%). Com exceção de um caso, todas as metástases foram identificados por tomografia computadorizada ou a cintilografia óssea. Tendo em conta os subtipos, como já esperado as metástases foram mais frequentes em Triplos Negativos (7,9\% de todos os tumores negativos) e Her2 (5,9\%), comparativamente a Luminal A $(4,2 \%)$ ou B (3,7\%), entretanto esta diferença não foi significativamente estatística. Após análise multivariada, estadio II foi o único fator independente para diagnóstico de metástase.

Conclusões: Este estudo corrobora as conclusões anteriores e indica que a baixa taxa de metástase detectável através do estadiamento radiológico inicial parece não justificar sua adoção na prática clínica para pacientes assintomáticos com câncer de mama precoce. Esta prática pode gerar custos elevados para os centros de tratamento ao câncer, além de submeter a paciente com câncer de mama a procedimentos estressantes sem benefício comprovado, de modo que se deve pesar a relação custo-benefício da sua adoção no estabelecimento de protocolos de conduta e na prática clínica diária. 